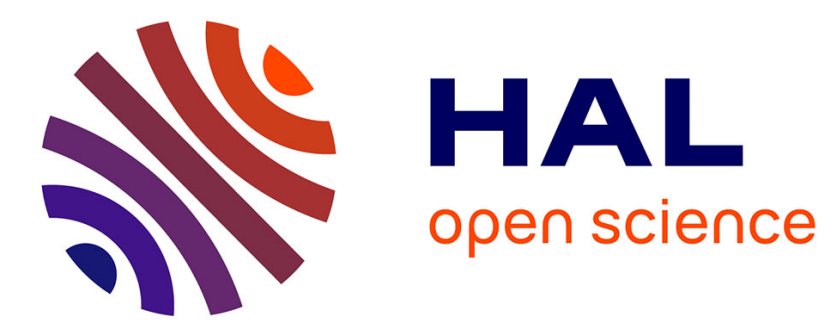

\title{
Binary pattern codification strategies in an active stereoscopic system based on flexible image guides
}

Erwan Dupont, Yingfan Hou, Frédéric Lamarque, Tanneguy Redarce

\section{To cite this version:}

Erwan Dupont, Yingfan Hou, Frédéric Lamarque, Tanneguy Redarce. Binary pattern codification strategies in an active stereoscopic system based on flexible image guides. SPIE Photonic West, Feb 2013, San Francisco, CA, United States. pp.86180H, 10.1117/12.2003601 . hal-00800000

\section{HAL Id: hal-00800000 https://hal.science/hal-00800000}

Submitted on 25 Apr 2019

HAL is a multi-disciplinary open access archive for the deposit and dissemination of scientific research documents, whether they are published or not. The documents may come from teaching and research institutions in France or abroad, or from public or private research centers.
L'archive ouverte pluridisciplinaire HAL, est destinée au dépôt et à la diffusion de documents scientifiques de niveau recherche, publiés ou non, émanant des établissements d'enseignement et de recherche français ou étrangers, des laboratoires publics ou privés.

$$
\text { Copyright }
$$




\title{
Binary pattern codification strategies in an active stereoscopic system based on flexible image guides
}

\author{
Erwan Dupont ${ }^{\mathrm{a}}$, Yingfan $\mathrm{Hou}^{\mathrm{a}}$, Frederic Lamarque ${ }^{\mathrm{a}}$, Tanneguy Redarce ${ }^{\mathrm{b}}$ \\ ${ }^{a}$ Université de Technologie Compiègne (UTC), Laboratoire Roberval UMR 7337, 1 rue du Dr \\ Schweitzer 60200 Compiègne, France \\ ${ }^{\mathrm{b}}$ Institut National des Sciences Appliquées de Lyon (INSA-Lyon), Laboratoire Ampère UMR 5005, \\ 20 av Albert Einstein 69100 Villeurbanne, France
}

\begin{abstract}
A wide variety of three dimensional (3D) measurement systems that can extract shape information's with sub millimetric accuracy is available in the industry. However, they generally are of macroscopic size and measuring on confined areas is not feasible. To miniaturize such systems, the step proposed is the integration of flexible image guides combined with compact optical probes.

This miniaturization process is tested on an active stereoscopic measurement system. In the projection channel of the system, a digital micro-mirror device (DMD) generates structured binary patterns from an incoherent white light source and injects them into a first image guide. Then, a compact optical system projects the pattern on the measurement area. The same configuration principle is applied to the acquisition channel and allows the capture of the measurement area through a second image guide and finally to a digital camera.

In this miniaturized system, image guides have lower resolution than in standard imaging devices. Indeed they are equivalent of 70k pixels devices to compare to the almost $800 \mathrm{k}$ pixels of the DMD and camera. That implies lower axial and lateral resolutions and consequently the shape reconstruction method must be carefully chosen. In this paper, several reconstruction strategies such as tuning the projected patterns frequency and also phase-shift versus gray code based methods were compared considering the best axial resolution criteria.
\end{abstract}

Keywords: 3D measurement - Image guides - Miniaturized probe - Time-multiplexed algorithms based on binary patterns - Active Stereoscopy - Digital micro-mirror device (DMD) - Micro scaled

\section{INTRODUCTION}

Three dimensional (3D) measurements are widely used in the industry for various purposes: reverse engineering process, quality control, structure deformation measurement.... In constrained areas, such as internal structures of mechanical systems, a miniaturized design of the device must be considered to help the 3D measurement. A way to successfully realize this is to move away the cumbersome components of the instrument by the use of fiber-optic. However this fiberoptics generally cause a drop in the depth resolution of the $3 \mathrm{D}$ reconstruction ${ }^{1,2}$. Then, in the case of such compact active stereoscopic devices, the choice of the projected patterns and the reconstruction algorithms are critical because the miniaturized optical components can generate a blurred effect on the captured result which can be compared to a defocus effect in a high resolution macro-scaled imaging system.

At macro scale, the defocus effect on the projected pattern is not always considered as a drawback but can rather be taken as an advantage to accurately build a well shaped sinusoidal pattern from a binary one ${ }^{3,4,5}$. Then a phase-shift algorithm is applied on the captured images to reconstruct the 3D-shape of the object. To obtain this result, the widths of black and white stripes of the binary pattern are modulated in a method called sinusoidal pulse width modulation (SWPM). This kind of method is suitable for high frequency measurements. Gong et al generate sinusoidal pattern at $4000 \mathrm{~Hz}$ using a DLP (Digital Light Processing, Texas Instrument Inc.) projector and a high speed CMOS camera to reconstructs the shape of a rotating fan blade ${ }^{6}$. 
The spatial frequency of the projected patterns has an impact on the quality of the reconstruction result. Varying this

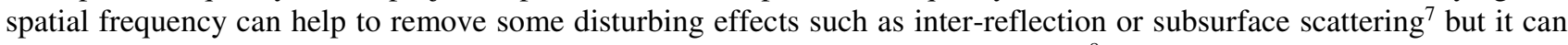
also increase the reconstruction performance in a blurred environment. Gupta et $\mathrm{al}^{8}$ proposes several sets of patterns at various frequencies, which are built using simple logical operations and tools from combinatorial mathematics.

In this paper, a miniaturized 3D stereoscopic system is presented based on a DMD which gives flexibility in the projecting patterns, and also based on image guides that are the main step towards compactness of the measuring probe. With the decrease of imaging resolution due to miniaturization, several patterns strategies are compared and their impact on the 3D reconstruction accuracy is experimentally demonstrated.

\section{MINIATURIZED STEREOSCOPIC SYSTEM DESCRIPTION}

To reconstruct the 3D shape, the proposed system is based on a miniaturized active stereoscopic device that project structured white light patterns. This device holds two compact measuring probes that are optically coupled to a camera and a projector through image guides as presented in figures 1 and 2.

The first part of the system is the projection channel that takes advantage of a DMD, which is a MEMS device composed of $1024 \times 768$ squared mirrors of $10.8 \mu \mathrm{m}$ pitch. Each micro-mirror of the DMD can be tilted in two discrete angles at a $32 \mathrm{kHz}$ rate. Only one of these two angles is optically active and allows the propagation of the light into the following optical system. When the micro-mirror is in the other angular position the corresponding pixel in the projected pattern is dark. With this principle, the DMD structures the incoming light into a $1024 \times 768$ pattern and injects it into an image guide which is coupled to a $6 \mathrm{~mm}$ diameter Steinhel triplet lens. This lens and an additional diaphragm compose the projection compact probe. The Steinhel triplet lens proved during optical simulation to be optimal for geometric and chromatic distortion on this miniaturized configuration.

The second part of the system is the acquisition channel. A second compact probe, with the same layout as the first one, captures an image of the measure area and injects it into a second image guide. Then the image is finally focused through an optical interface to a CCD sensor composed of $1024 \times 768$ pixels. There is a stereoscopic angle of $60^{\circ}$ between the projection and the capture channels. Using the triangulation principle, this stereoscopic angle encodes the depth information of the measured surface. With a high stereoscopic angle, the depth resolution increases, but the risk to have occluded areas, where the measure is not possible, also increases.

The 2 image guides included in the 3D measuring system are optical components that copy an image from one of their end to their other end. Each image guide is composed of 70000 optical fibers of $4 \mu \mathrm{m}$ diameter each. They length is 2 meters and diameter is $1.4 \mathrm{~mm}$. In terms of image resolution, the image guide is the bottleneck of the system. Indeed, camera and DMD are equivalent of 800000 pixels imaging devices which has to be compare to the 70000 pixels of the image guide. The low resolution of the image guides directly impacts the depth resolution of the instrument; however by decreasing the field of view of the capturing and projecting channels, the depth resolution can be improved.

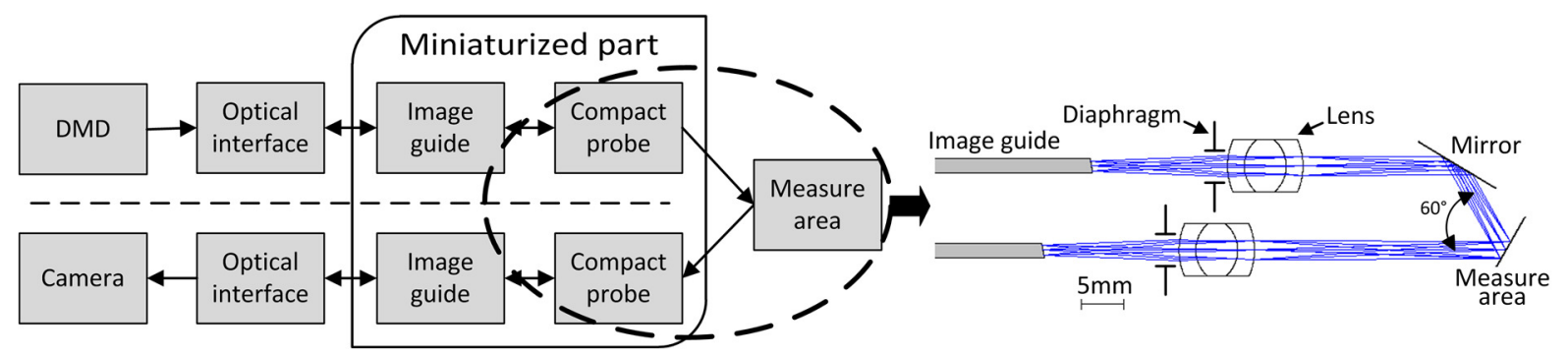

Figure 1: Schematic representation of the 3D measurement system. 


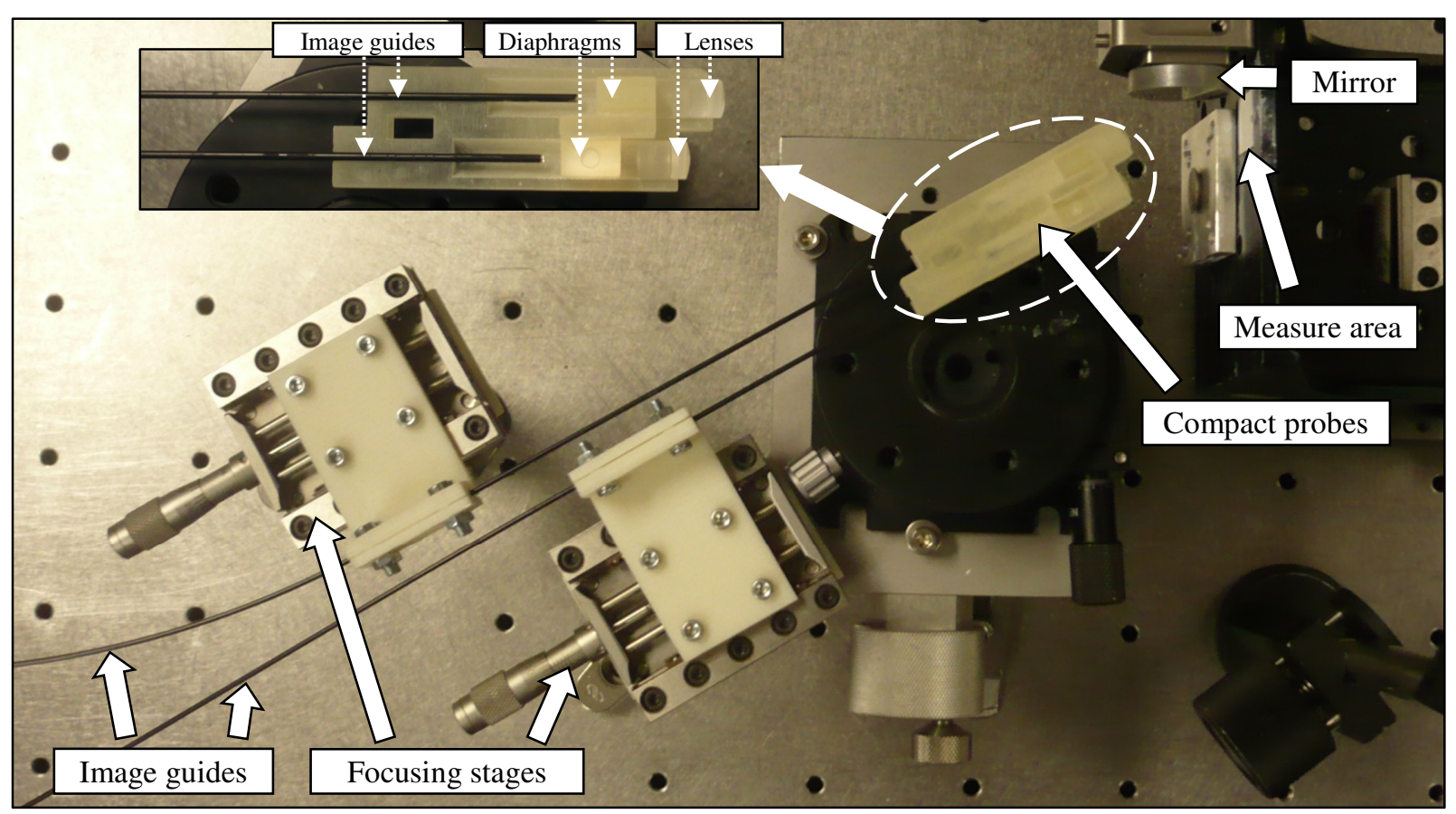

Figure 2: Photography of the 3D measurement system

\section{PROPOSED RECONSTRUCTION METHODS}

\subsection{Time multiplexing method versus other methods}

Some reviews ${ }^{9,} 10$ have clearly organized the structured light projection techniques. A classification in three global principles is proposed:

The first one is the spatial neighborhood principle, where generally a unique pattern is projected onto the measure area. With this principle, the analysis of the pattern that surrounds a pixel gives the codeword that label the pixel. As there is only one projected pattern, the spatial neighborhoods method can be used to record dynamic scenes; however it can be hard to accurately detect the shape of the illuminated object when there are shadows and occlusions on the object. Shadows and occlusions generate holes in the captured pattern and can lower the quality of the decoding process on their neighborhood.

The second principle is the direct codification strategy. Each pixel of the projected pattern contains a specific color or grey level. This specific information is then detected and identifies the corresponding projected pixel. This method is very sensitive to the noise on the measured surface and generally requests to capture more than one image of the scene, which is not suitable in dynamic environments. To get a higher resolution result with this direct codification strategy, the projected pattern is generally periodic.

The third principle of the 3D structured light reconstruction methods that gives the highest resolution results is the time multiplexing principle. A set of patterns are successively projected onto the measure area and the codeword for one pixel is given by the intensity variation between the sequences of values received by the pixel. With a high number of projected patterns, the position of one pixel will be more accurately detected and the resolution of the measurement will increase. The main constraint of these methods based on time multiplexing is that they are inapplicable to dynamic scenes. In a microscopic context where the scenes to be measured are mainly static, the time multiplexing methods can be the ones with the best resolution results.

In the time multiplexing methods scope, some methods are based on binary codes, where black and white patterns are successively projected onto the surface. Other methods use phase-shifting where a periodic pattern is projected onto the 
measured surface and then shifted by small increments. The binary code and phase-shift methods are both possible solutions on the proposed device. Two methods based on binary codes and two methods based on phase-shift have consequently been selected and their performance evaluated.

\subsection{Distinction criteria between the 4 methods}

As stated above, four methods are experimentally tested in this work. Two of these methods are gray code based, with different spatial frequencies for the projected patterns: a classical gray code with some high frequency patterns and an optimized gray code with low frequency pattern (as low as possible). The other two methods are based on phase-shift reconstruction methods from blurred binary patterns. The phase shift methods are tested for two pattern frequencies.

Every projected pattern will have a width of 1024 pixels, which is the horizontal size of the DMD. A criterion used to evaluate the different algorithms was their resolution (8,9 and 10 bits were tested). A second criterion was the projected patterns frequency which can be measured towards the "minimum stripe width" (Min-SW).

The highest spatial frequency contained in a pattern is measured with the minimum stripe width. For example, in a classical binary code of 10 bits (10 patterns), where the values goes from 0 to 1023, stripe width on the first one (MSB) of the 10 patterns is 512 , stripe width on the second one is 256 , etc..., and stripe width on the last pattern (LSB) is 1 . Consequently, a classical 10 bit binary code has a Min-SW of 1. With the proposed 3D measurement system, a Min-SW of 1 is too small, the frequency of the patterns will be too high and not detected, black and white patterns will both be gray after the capture without contrast between them. As a consequence, due to the low resolution of the optical system, the frequency of the projected patterns must be as low as possible to easily detect the signal variations and the Min-SW must be maximized.

\subsection{Classical gray code patterns}

The first set of patterns tested on the measuring system is gray code based. Gray code, also known as reflected binary code, is a coding system where two successive values differ in only one bit. A classical gray code pattern is presented in figure 3(a). This coding principle has the advantages to make the patterns more robust to noise and also allows error correction after the capture process by checking that only one bit has changed between two successive values.

In our projection setup, the size of the pattern that the DMD can project is 1024 . If 10 bits gray code (1024 values) is projected, with 10 patterns, the Min-SW of the projected pattern will be of 2, instead of 1 with classical binary code. If 9 bits gray code (512 values) is projected, so with 9 patterns, the Min-SW of the projected pattern will be of 4 , the 512 values being encoded over the 1024 horizontal pixels of the DMD. And finally with an 8 bits gray code, and 8 patterns, the Min-SW will be of 8, with 256 values encoded over the 1024 horizontal pixels of the DMD.

The classical gray code tested on the system will be of 8, 9 and 10 bits, with a Min-SW of respectively 8, 4 and 2 .

(a)

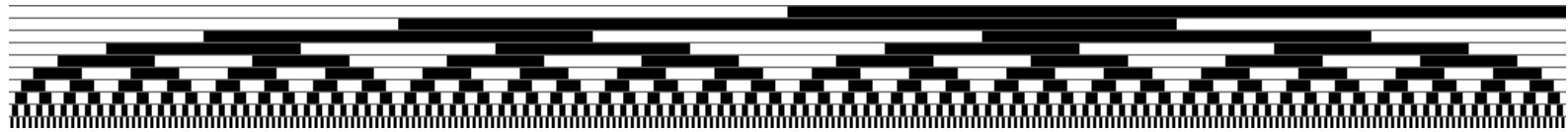

(b)

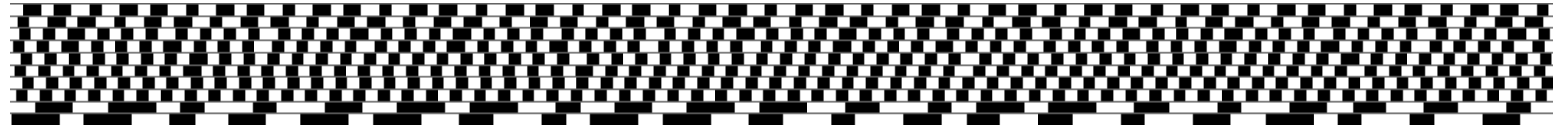

(c)

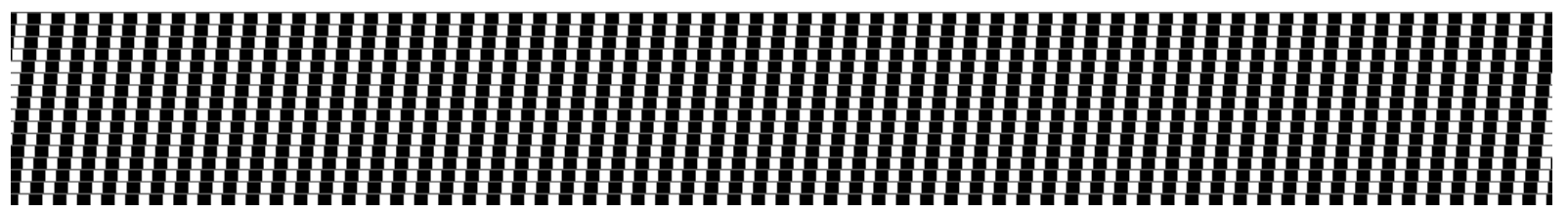

Figure 3: (a) The 10 patterns for the 10 bits classical gray code method (b) The 10 patterns for the 10 bits MMSW method (c) The 16 patterns for the 10 bits phase shifting (classical and alternative) algorithms with 8 pixels stripe width 


\subsection{Maximum Min-SW binary patterns}

Some specific binary codes are optimized for high Min-SW and used for 3D reconstruction ${ }^{10}$. Although these kinds of codes are rare, they have been well studied in the combinatorial mathematics ${ }^{11}, 12$. One of these codes, shown in figure 3(b), will be tested with the proposed device. For this maximum Min-SW (MMSW) binary method, no generic algorithm allows building $n$ bits patterns, for any value of $n$. However, for $n=10$, an algorithm is proposed and well detailed ${ }^{11}$.

The minimum stripe width of this 10 bits MMSW code is of 8 . So if 10 bits MMSW code is projected, with 10 patterns, the Min-SW of the projected pattern will be of 8 with 1024 distinct values encoded on the 1024 horizontal pixels of the DMD. To obtain a 9 bits MMSW code (so with 512 values), the 10 bits MMSW code is used with its 10 patterns, but only the first half of the 1024 values is projected. Consequently, if 9 bit MMSW code is projected, with 10 patterns, the Min-SW of the projected pattern will be of 16, with 512 distinct values encoded on the 1024 horizontal pixels of the DMD. With the same reasoning for an 8 bit MMSW code, with 10 projected patterns, the Min-SW will be of 32 with 256 values encoded on the 1024 horizontal pixels of the DMD.

The MMSW code tested on the system will be of 8, 9 and 10 bits, with a Min-SW of respectively 32, 16 and 8 .

In figure 3, the classical gray code (a) has a Min-SW of 2 and the MMSW code (b) has a Min-SW of 8. It is obvious on the images that the classical gray code has higher frequency stripe width than MMSW that keeps the frequency as low as possible over the 10 patterns. But the quantity of distinct information encoded in the patterns is the same for the two codes, and equal to 1024 .

\subsection{Phase-shift patterns}

Two phase-shift algorithms are also tested; which both use the same set of patterns. An example of these patterns is presented in figure 3(c). The two phase-shift algorithms are based on different principles: for the first one, the binary pattern is assumed to become sinusoidal after projection, due to blurring effect. The second algorithm doesn't assume a sinusoidal response and fit more precisely the shape of the captured signal.

The Min-SW for the phase-shift patterns is equal to the width of the black or white stripes. The resolution of the measurement is linked to the number of pixel for one step. For example, if the patterns have a stripe width of 16 pixels, and if 32 shifts of 1 pixel are done, then 1024 distinct values will be encoded. Consequently the resolution of the projected patterns will be of 10 bits $\left(2^{10}=1024\right)$. On the other hand, if there are only 16 patterns shifted of 2 pixels from one pattern to the other, then 512 values will be encoded on the 1024 width of the projection device, the resolution of this phase-shift will be of 9 bits $\left(2^{9}=512\right)$.

For the first phase-shift method, a classical phase-shift algorithm is applied. Binary patterns such as the ones described in figure 3(c) are projected onto the measure area. The captured patterns are considered to be sinusoidal and the observed intensity $\mathrm{I}_{\mathrm{i}}$ onto a pixel of the camera follows the formula

$$
\mathrm{I}_{\mathrm{i}}(\mathrm{x}, \mathrm{y})=\mathrm{L}_{1}(\mathrm{x}, \mathrm{y}) \times \cos \left(\phi(\mathrm{x}, \mathrm{y})+\delta_{\mathrm{i}}\right)+\mathrm{L}_{2}(\mathrm{x}, \mathrm{y})
$$

Where $\mathrm{x}$ and $\mathrm{y}$ are the coordinates of the pixel onto the captured image. $\mathrm{L}_{1}(\mathrm{x}, \mathrm{y})$ and $\mathrm{L}_{2}(\mathrm{x}, \mathrm{y})$ are light intensity, that depend of the illumination of the scene (global and direct illumination). $\delta_{\mathrm{i}}$ is the phase-shift for the $\mathrm{i}^{\text {th }}$ pattern. Finally, $\phi(x, y)$ is the observed phase and is correlated to the depth information. It depends on the specific projection and capture devices parameters. The observed phase can be calculated:

$$
\phi(\mathrm{x}, \mathrm{y})=\tan ^{-1}\left(\frac{-\sum \mathrm{I}_{\mathrm{i}}(\mathrm{x}, \mathrm{y}) \sin \left(\delta_{\mathrm{i}}\right)}{\sum \mathrm{I}_{\mathrm{i}}(\mathrm{x}, \mathrm{y}) \cos \left(\delta_{\mathrm{i}}\right)}\right)
$$


For $\mathrm{N}$ spaced phase shifts, the sums of equation 2 are over the $\mathrm{N}$ measurements. $\phi(\mathrm{x}, \mathrm{y})$ is in the range of $[-\pi ;+\pi]$, and after a phase unwrapping process, it can be scaled on the same $[0 ; 1023]$ range than the classical gray and MMSW codes.

The second phase-shift algorithm, named alternative phase-shift, is detailed in another study ${ }^{13}$ and is based on the same set of patterns than the classical one. The signal captured by each pixel of the camera is represented with an intensity function $F(t)$ (equivalent as $I_{i}(x, y)$ on the previous phase-shift algorithm). As presented in figure 4, this intensity function is calculated with the convolution of the binary projected pattern function $\mathrm{G}(\mathrm{t})$ and the blurred effect function $\mathrm{H}(\mathrm{t})$

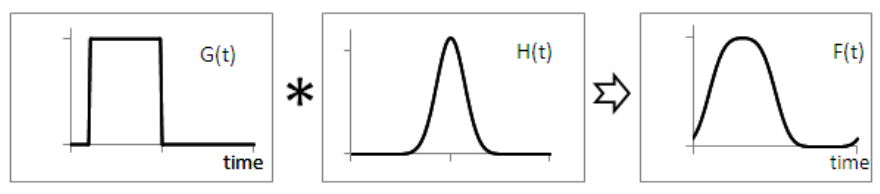

Figure 4: Calculation of the captured signal $\mathrm{F}(\mathrm{t})$

The function $\mathrm{H}(\mathrm{t})$ is equivalent to the blurred effect due to the various distortion and diffraction effects in the optical system:

$$
H(t)=\frac{1}{\sigma \sqrt{\pi}} \exp \left(-\left(\frac{x}{\sigma}\right)^{2}\right)
$$

The blurred effect in the whole optical system is identified with respect to measurement to set the value of the $\sigma$ parameter. Then, a Levenberg-Marquardt Algorithm (LMA) is applied on the intensity function measured on each pixel of the camera to determine the observed phase $\phi(x, y)$. After a phase unwrapping process, $\phi(x, y)$ is scaled on the $[0 ; 1023]$ range. The advantage of this alternative phase-shift algorithm compared to the first one is that it can precisely fit non-sinusoidal captured signal, especially in case of low blur in images.

For each one of the two phase-shift algorithms, 8,9 and 10 bits patterns are projected; shifts of the pattern are respectively of 4, 2 and 1 pixel(s). And these projections are tested with patterns of Min-SW equal to 8 and 16.

\section{EXPERIMENTAL RESULTS}

The experimentations are done with the 3D stereoscopic measurement device described in part 2 and tested with the four methods proposed in part 3, and with 3 resolutions ( 8,9 and 10 bits) for each method. For the two phase-shift methods, two distinct stripe widths (equal to the min-SW) of 8 and 16 pixels are tested. The measurements are done on a white flat surface and also on a 10 cent euro coin as shown in figure 5.

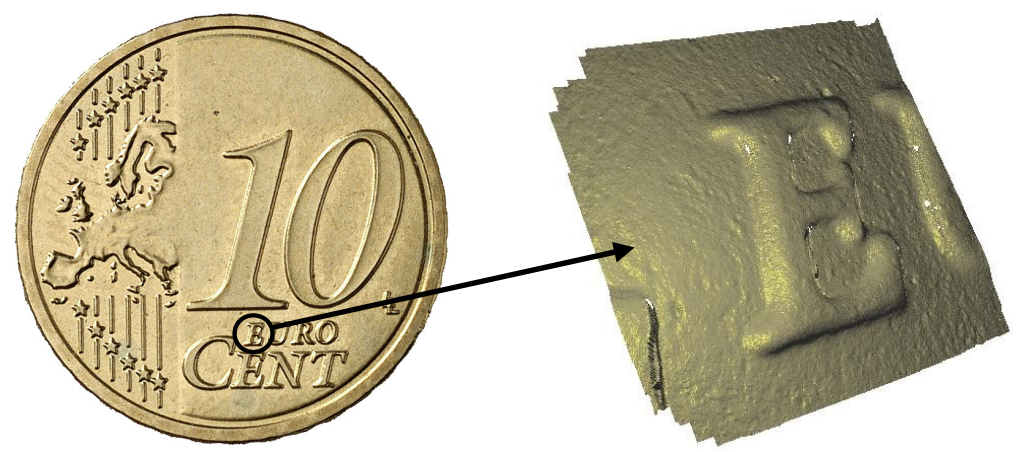

Figure 5: The E letter of the EURO word is reconstructed from 10 cents of euro coin 
For each pattern that needs to be projected on the object, two patterns are actually projected: the intended one and its opposite (black pixels become white and vice versa). The two captured image are then subtracted to obtain one differential final image. This process allows eliminating the background illumination and other sources of noise. It gives more accurate results, with the drawback of an increase of the number of projected patterns.

For all the pixels of one set of projected patterns, the intensity is computed to get one phase value per pixel. This phase value is converted to height values and the pixel coordinates are also converted to real word coordinates. During the reconstruction process, no low pass spatial or temporal filtering is applied to the raw data in order to compare on the same basis the different algorithms. Some results with phase and depth values measured on the 10 cent euro coin with the 8 bits gray-code and 8 bits phase-shift method is presented in figure 6.

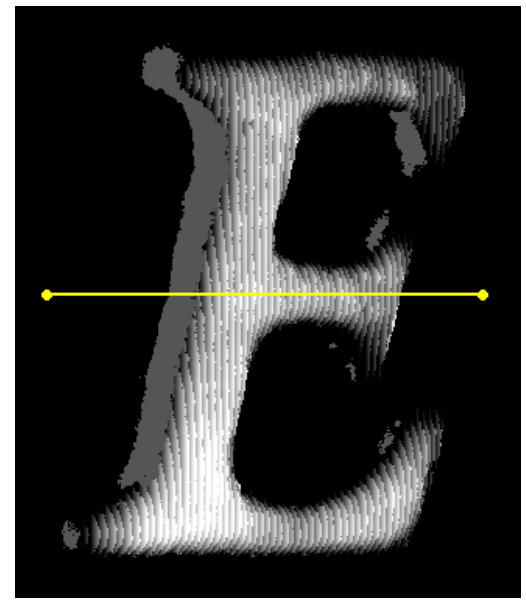

(a)

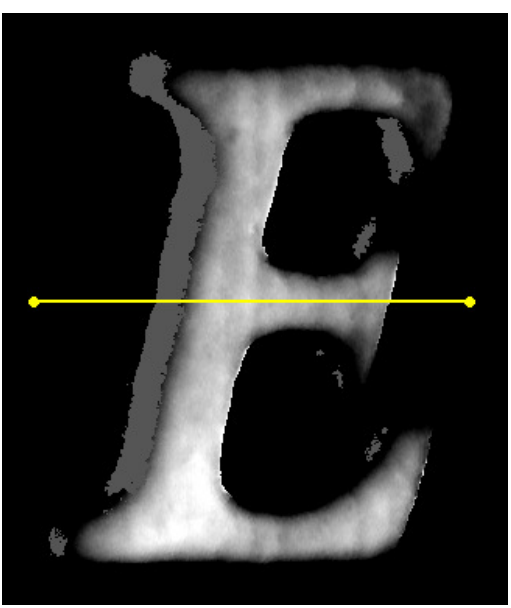

(c)
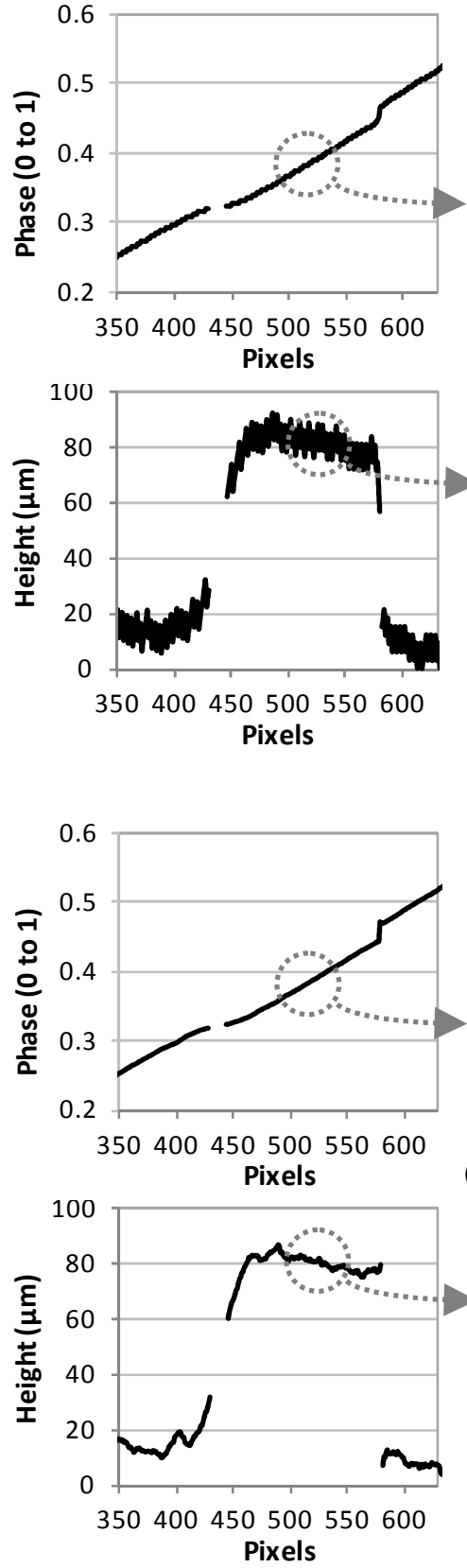

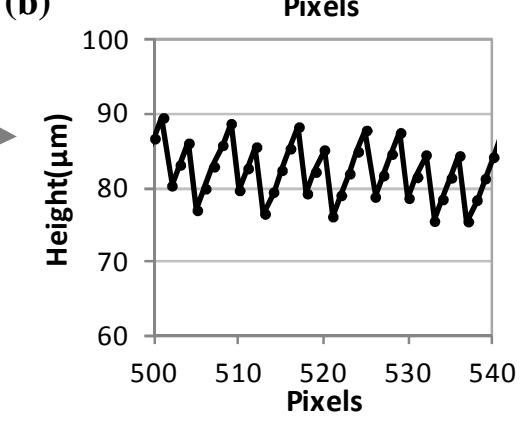

(d)
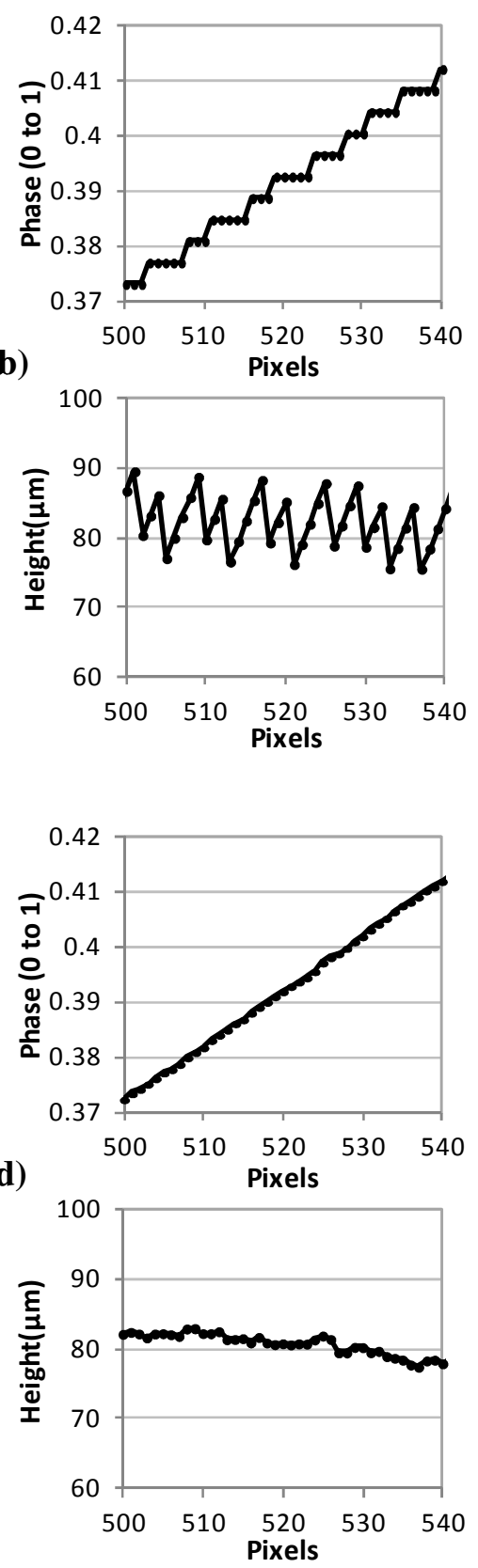

Figure 6: (a) Depth map of the E letter of the 10 cent euro coin reconstructed with a 8 bits Gray code algorithm (b) Phase and depth profiles over a line of the E letter with a 8 bits Gray code algorithm (c) Depth map of the E letter reconstructed with a 8 bits Phase-shift algorithm (d) Phase and depth profiles over a line of the E letter with a 8 bits Phase-shift algorithm 
For each algorithm, a profile along a horizontal line of the white flat surface is extracted from the depth map. Then the standard deviation of the measured height is calculated. The same method is also applied for a profile on the 10 cent euro coin. However, the standard deviation on the coin will not measure the noise around a single depth values but will rather measure the dispersion of the depth values due to the varying shape of the coin. To measure the noise due to the reconstruction process, it is assumed that the shape of the coin is a low frequency signal and consequently a high pass filter is applied on the depth profile. The method to apply this filter is presented in figure 7.

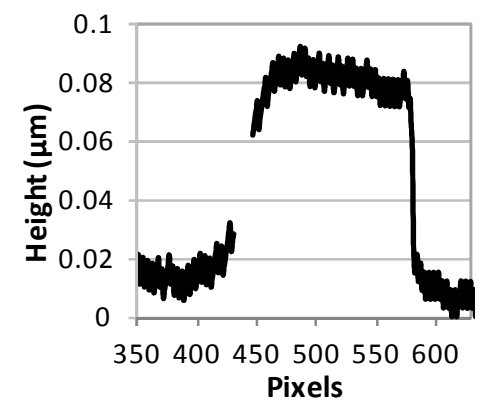

(a)

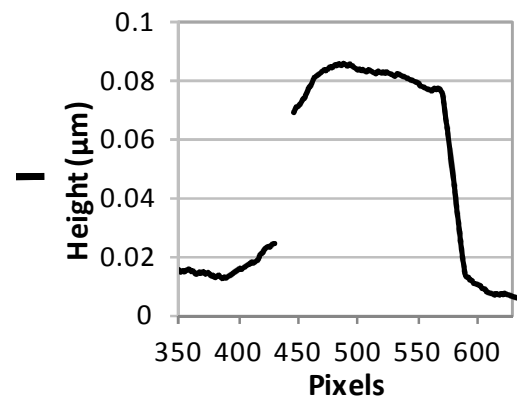

(b)

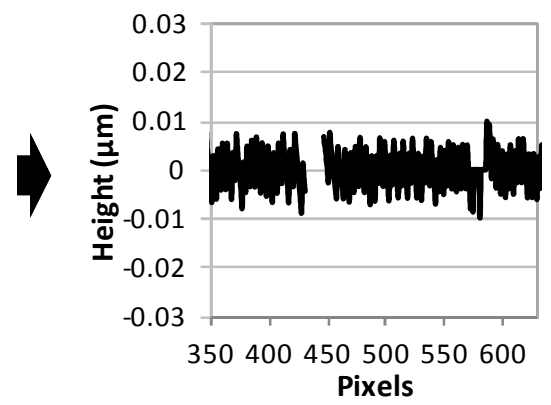

(c)

Figure 7: High pass filter method : (a) profile on a 10 cent euro coin with a 8 bits gray code algorithm (b) low pass filter on the measured profile (c) subtraction of the raw profile with the low pass filtered profile to obtain the high pass filtered profile

Once the measure is high pass filtered, the standard deviation is computed and gives an estimation of the noise due to the reconstruction process. This high pass filtering process is applied on the profile extracted from the 10 cent euro coin. Tables 1, 2 and 3 present the measurement results for the four algorithms, and in 3 different resolutions for each algorithm. The influence of the high pass filter on the final standard deviation can be visualized on the 3 tables in case of white flat surface illumination because the calculations are done with and without the use of the filter.

\begin{tabular}{|c|c|c|c|c|c|c|c|}
\cline { 3 - 8 } & \multicolumn{2}{c}{} & \multicolumn{2}{c|}{ Gray code method } & \multicolumn{3}{c|}{ MMSW code method } \\
\cline { 2 - 8 } & Projection resolution (bits) & $\mathbf{8}$ & $\mathbf{9}$ & $\mathbf{1 0}$ & $\mathbf{8}$ & $\mathbf{9}$ & $\mathbf{1 0}$ \\
\cline { 2 - 8 } & Min stripe width (Min-SW) & 8 & 4 & 2 & 32 & 16 & 8 \\
\hline \multirow{2}{*}{ White flat surface } & Number of images & 8 & 9 & 10 & 10 & 10 & 10 \\
\hline & Depth standard deviation $\sigma(\mu \mathrm{m})$ & 3.71 & 3.74 & 4.10 & 3.62 & 2.09 & 1.96 \\
\hline $\mathbf{1 0}$ cent euro coin & Depth $\sigma(\mu \mathrm{m})$ (high pass filtered) & 3.66 & 3.58 & 3.79 & 3.66 & 2.03 & 1.92 \\
\hline
\end{tabular}

Table 1: Measurement results on a white flat surface and on a coin with the gray code and MMSW method

\begin{tabular}{|c|c|c|c|c|c|c|c|}
\cline { 3 - 8 } & \multicolumn{5}{c}{} & \multicolumn{5}{c|}{ Classical phase-shift algorithm } \\
\cline { 2 - 9 } & Projection resolution (bits) & $\mathbf{8}$ & $\mathbf{9}$ & $\mathbf{1 0}$ & $\mathbf{8}$ & $\mathbf{9}$ & $\mathbf{1 0}$ \\
\cline { 2 - 9 } & Min stripe width (Min-SW) & 8 & 8 & 8 & 16 & 16 & 16 \\
\hline & Number of images & 4 & 8 & 16 & 8 & 16 & 32 \\
\hline \multirow{2}{*}{ White flat surface } & Depth standard deviation $\sigma(\mu \mathrm{m})$ & 2.06 & 1.61 & 1.44 & 1.04 & 0.96 & 0.82 \\
\hline & Depth $\sigma(\mu \mathrm{m})$ (high pass filtered) & 2.02 & 1.50 & 1.33 & 1.08 & 0.95 & 0.91 \\
\hline 10 cent euro coin & Depth $\sigma(\mu \mathrm{m})$ (high pass filtered) & 2.33 & 2.07 & 1.96 & 1.69 & 1.65 & 1.65 \\
\hline
\end{tabular}

Table 2: Measurement results on a white flat surface and on a coin with the classical phase-shift algorithm 


\begin{tabular}{|c|c|c|c|c|c|c|c|}
\hline & \multirow[b]{2}{*}{ Projection resolution (bits) } & \multicolumn{6}{|c|}{ Alternative phase-shift algorithm } \\
\hline & & 8 & 9 & 10 & 8 & 9 & 10 \\
\hline & Min stripe width (Min-SW) & 8 & 8 & 8 & 16 & 16 & 16 \\
\hline & Number of images & 4 & 8 & 16 & 8 & 16 & 32 \\
\hline \multirow{2}{*}{ White flat surface } & Depth standard deviation $\sigma(\mu \mathrm{m})$ & 1.20 & 1.06 & 1.00 & 0.73 & 0.69 & 0.68 \\
\hline & Depth $\sigma(\mu \mathrm{m})$ (high pass filtered) & 0.84 & 0.78 & 0.60 & 0.63 & 0.59 & 0.57 \\
\hline 10 cent euro coin & Depth $\sigma(\mu \mathrm{m})$ (high pass filtered) & 1.64 & 1.59 & 1.56 & 1.36 & 1.35 & 1.34 \\
\hline
\end{tabular}

Table 3: Measurement results on a white flat surface and on a coin with the alternative phase-shift algorithm

In any cases, phase-shift algorithms give more precise results than gray code and MMSW methods. However it must be noted that phase-shift algorithms requires a phase unwrapping process (to resolve the ambiguity on the phase) that is not needed in the two other methods. This phase unwrapping process can be resolved with additional images projection (with the cost of additional capture time), or with specific algorithms (with the cost of additional computation time).

Except the classical gray code, all the algorithms have a decreasing standard deviation when the resolution increases. The classical gray code doesn't give a decreasing standard deviation for an increase of the resolution, because its MinSW values are too low (4 and 2). The blurring effect due to the miniaturized optical systems doesn't allow detecting stripes width below 8 pixels. If the stripes spatial frequency is below this limit, the contrast between the projected black and white stripes in the final captured image isn't sufficient. Consequently the MMSW codes or others high Min-SW codes (such as antipodal gray codes ${ }^{12}$ ) must be preferred to the classical Gray code in a blurred or defocused environment due to their optimized Min-SW value. The same reasoning can be applied on the phase-shift algorithms. Standard deviation measured that have a Min-SW of 16 gives better results than the one measured with a Min-SW of 8 . Consequently, in miniaturized optical systems that has a low imaging resolution (in this experimental case, due to the 70000 fibers image guides) the resolution of the algorithm $(8,9$ or 10 bits for example) is a criterion that fix the quality of the reconstructed shape, but the Min-SW of the projected pattern is also important.

The best result is obtained with the alternative phase-shift algorithm applied to a white flat surface, a 10 bits algorithm and with a Min-SW of 16 . Indeed the calculated standard deviation is of $0.68 \mu \mathrm{m}$. However, $32 \times 2$ patterns must be projected that implies an increase of the capture time. The 9 bits algorithm gives almost the same measurement results with a half quantity of patterns $(16 \times 2)$. Although the alternative phase-shift algorithm gives better result than the classical one, it must be noted that the alternative phase-shift algorithm is based on a LMA and its computation time for the reconstruction is higher.

To conclude this analysis, if the computation time is critical, the MMSW method will give good results with 10 captured pattern and 9 or 10 bits of resolution. If the depth resolution is the main parameter to optimize, the phase-shift algorithms must be preferred, with the necessity of an additional phase unwrapping process. In this goal, the classical phase-shift algorithm provide fast computation (but slower than MMSW) while the alternative phase-shift is more optimized for high precision measurement.

\section{CONCLUSION}

A 3D shape measurement system with a compact design is presented in this paper. To obtain this miniaturized setup, 2 meters length image guides with $1.4 \mathrm{~mm}$ diameters and resolution of 70000 pixels are used for both acquisition and projection channels. These image guides have the advantages to reduce the volume of the instrument near the measure area and allows transmitting images over long distance (up to 10 meters) with the drawback of lowering the imaging resolution.

Projected patterns and reconstruction algorithms must be adapted to this low imaging resolution. Time multiplexing strategies for the pattern projection were chosen for their high accuracy with the downside of capturing only static scenes. Considering these time multiplexing strategies, the phase-shift methods proved to give more precise 3D reconstruction and the gray code based methods have the fastest reconstruction time. A critical criterion for the choice of 
the reconstruction method is the spatial frequency of the projected pattern. In a low resolution imaging system, this spatial frequency must not be too high otherwise the contrast between the dark and bright stripes of the captured patterns will not be sufficient; The selected methods must use patterns with a high "minimum stripe width" (min-SW). A gray code method based, the maximum min-SW method (MMSW), proved to give better 3D reconstruction than the classical gray code method. In addition, phase-shift patterns with a Min-SW of 16 prove to be more optimized.

On a metrology viewpoint, the experimentations showed that the depth resolution of the 3D measuring system is near the micrometer, with a standard deviation of less than $1 \mu \mathrm{m}$ on a measured white flat surface. The lateral resolution was estimated around $25 \mu \mathrm{m}$ for a field of view of $3 \mathrm{~mm}$ square. The depth of field for this measurement was estimated around $1 \mathrm{~mm}$.

Future works will seek to optimize the depth resolution of the current optical miniaturized system by studying a zoom effect caused by the variable optical magnification of the imaging system. This variable optical magnification is obtained with slight changes on the position of the triplet Steinhel and diaphragm with respect to the two images guides. Due to this magnification, the field of view is reduced but the axial and lateral resolution can be increased, till the diffraction limit. Further miniaturization process is also planned on the current system with the use of gradient-index (GRIN) lenses instead of the triplet Steinhel lenses. Their impact on the measurement resolution has to be evaluated.

\section{REFERENCES}

[1] Schmalz C., Forster F, Schick A., Angelopoulou E. “An endoscopic 3D scanner based on structured light”, Medical Image Analysis, Volume 16, Issue 5, pp 1063-1072, (2012)

[2] Fan K.; Li R., Song H., "Fibre image techniques in digital stereomicroscopy", Measurement Science and Technology, Vol. 17, pp. 373-378, (2006)

[3] Serrano-Heredia A, Hinojosa C, Ibarra J. \& Arrizon V., "Recovery of three-dimensional shapes by using a defocus structured light system”, Proc. SPIE 3520, Three-Dimensional Imaging, Optical Metrology, and Inspection IV, 80 (1998)

[4] Lei S. \& Zhang S., "Flexible 3-D shape measurement using projector defocusing", Optics letters, Vol. 34, Issue 20, pp. 3080-3082 (2009)

[5] Zuo C., Chen Q., Feng S., Feng F., Gu G. \& Sui X., "Optimized pulse width modulation pattern strategy for threedimensional profilometry with projector defocusing”, Applied Optics, Vol. 51, Issue 19, pp. 4477-4490 (2012)

[6] Gong Y. \& Zhang S, "Ultrafast 3-D shape measurement with an off-the-shelf DLP projector", Optics Express, Vol. 18, Issue 19, pp. 19743-19754 (2010)

[7] Gupta M., Yuandong Tian, Narasimhan S.G.; Li Zhang; , "(De) focusing on global light transport for active scene recovery", Computer Vision and Pattern Recognition, 2009. CVPR 2009. IEEE Conference on , pp.2969-2976, (2009)

[8] Gupta, M., Agrawal A., Veeraraghavan A., Narasimhan S.G., "Structured light 3D scanning in the presence of global illumination", Computer Vision and Pattern Recognition (CVPR), IEEE Conference, pp.713-720, (2011)

[9] Siva Gorthi S. \& Rastogi P., "Fringe projection techniques: whither we are?" Opt. Lasers Eng. 48(2), 133-140 (2010). 
[10] J. Salvi, J. Pagès, J. Batlle, "Pattern codification strategies in structured light systems", Pattern Recognition, Volume 37, Issue 4, April 2004, Pages 827-849, (1993)

[11] Goddyn L. \& Gvozdjak P., "Binary Gray Codes with Long Bit Runs", Electronic Journal of Combinatorics, Vol. 10, pp. 27 (2003)

[12] Kim D., Ryu M. \& Lee S., “Antipodal gray codes for structured lightRobotics and Automation”, 2008. ICRA 2008. IEEE International Conference, pp. 3016 -3021, (2008)

[13] Dupont E., Lamarque F., Prelle C. \& Redarce T., "Tri-dimensional optical inspection based on flexible image guide: first step toward 3d industrial endoscopy" Proceedings of ESDA, Vol. 82453, (2012) 